Methods Qualitative community based participatory research. Multiple recruitment strategies were used to purposively sample the British Bangladeshi population. Community facilitators were an active partner in this study and worked as a bridge to the community. A total of 145 participants completed the study and were recruited to 12 focus groups and 45 semi-structured interviews, including; British Bangladeshi mothers, fathers, grandmothers and grandfathers of infants and young children aged 6-23 months; key informants and lay community members from the British Bangladeshi population and health professionals. Transcripts were then checked and were analysed using framework analysis.

Results Two overarching themes were identified during interviews and group discussions: (1) modifiable infant feeding practices that participants suggested could be targeted in order to optimise infant feeding and (2) socioecological factors believed by participants to influence these modifiable feeding practices. Participants discussed several infant feeding practices that may restrict dietary diversity, override infant satiety and encourage dependence on sugary or sweetened foods and impact on broader care practices such as sleeping and physical activity. Specifically, four modifiable infant feeding and care practices were highlighted: untimely introduction of semi- and solid foods, overfeeding, prolonged parent-led feeding and feeding to 'fill the belly'. These practices were driven by factors at all socio-ecological levels, categorised as: society, culture and religion, physical and local environment and information and awareness.

Conclusions Parenting interventions need to be culturally sensitive to populations of diverse ethnicity and recognise the social and cultural norms that inform complementary feeding practices. Further, the infant feeding environment requires better regulation of marketing of foods for infant and young children. More evidence is needed to inform effective early years interventions.

\section{G165 HEALTH SERVICES FOR LONG TERM CONDITIONS FROM THE CHILD AND CAREGIVER'S PERSPECTIVE: A QUALITATIVE ANALYSIS}

${ }^{1} \mathrm{R}$ Satherley, ${ }^{2} \mathrm{~K}$ Ewan, ${ }^{2} \mathrm{~J}$ Green. ${ }^{1}$ Department of Women and Children's Health, King's College London, London, UK; ${ }^{2}$ Population Health and Environmental Sciences, King's College London, London, UK

\subsection{6/archdischild-2020-rcpch. 136}

Aims The current model of hospital-centred paediatric care in high-income countries was developed to deliver acute specialist services, rather than high quality care for children and young people (CYP) with long term conditions (LTCs) that require multidisciplinary, integrated care. While the limitations of current child health services in the UK have been widely documented from the perspectives of clinicians, academics and policy makers, there is a lack of research into the lived experience of CYP receiving these services. This qualitative study explores CYP and caregivers' experiences of health services for LTCs.

Methods An inductive, qualitative design using in-depth, semi-structured interviews with children (4-16 years; $n=20$ ) and their caregivers, receiving care for one of four LTCs (constipation, eczema, asthma or epilepsy). These LTCs were chosen as they are examples of LTCs which provide generalisable lessons about improving outcomes for other LTCs. Art-based methods were used to engage younger children in the discussions. Data was analysed using interpretive thematic analysis.

Results Families expressed several challenges associated with the healthcare they had received during the diagnostic process, including being unsure whether their symptoms warranted health intervention, a lack of specialist knowledge across primary care providers, and poor care continuity. After diagnosis, families desired further understanding around condition management, children wished to be included in discussions with health providers, and families felt there were no opportunities to discuss the psychosocial impact of the condition, particularly around the challenges managing health in school settings.

Conclusions Families reflected on the health services failures to address their needs. Psychosocial support, continuity of care and opportunities for independence play a critical role in promoting the health of children with LTC. These features are essential in the development of integrated care models, to ensure suitability of the workforce and improve care delivery.

\section{G166 SERVICE DESCRIPTION: AN INTEGRATED PATHWAY FOR UNACCOMPANIED ASYLUM SEEKING CHILDREN}

${ }^{1} \mathrm{AJ}$ Armitage, ${ }^{2} \mathrm{~J}$ Cohen, ${ }^{2} \mathrm{~S}$ Eisen, ${ }^{1} \mathrm{M}$ Heys, ${ }^{3} \mathrm{~A}$ Ward. ${ }^{1}$ Institute of Child Health, UCL, London, UK; ${ }^{2}$ Paediatric department, UCLH, London, UK; ${ }^{3}$ Camden Community Paediatrics, CNWL, London, UK

\subsection{6/archdischild-2020-rcpch.137}

Aims Unaccompanied asylum seeking children (UASC) in the UK have poor mental and physical health outcomes, and studies suggest that current services are failing to meet their needs. There is a lack of knowledge and expertise about UASC health management among health professionals and no standardised guidance exists for their care.

We describe a novel integrated pathway for UASC developed in a London borough and present data for a cohort of UASC receiving input from this service.

Methods

Service description: An integrated pathway for UASC was developed with a multi-disciplinary approach, coordinating input from the LAC team, primary care, infectious diseases (ID), sexual health (SH) services and CAHMS, and including a dedicated health improvement practitioner to ensure continuity and promote joined-up care.

Population: We reviewed UASC undergoing initial health assessments (IHAs) in this service between January 2016 and March 2019. Data were also collected on UASC referred from the same service to a local SH clinic and for ID screening. Results

- 101 UASC were seen for IHA (84\% male, age range 14-17, median age 16) in the integrated pathway model. Where documented, a translator was required for $87 / 90$ (97\%) of appointments.

- $92 \%$ of UASC were referred for ID screening, regardless of symptoms. 91\% were offered some form of CAMHS support: $51 \%$ were referred, 20\% declined referral and 20\% were signposted.

- $87 \%$ of UASC were referred or signposted to sexual health services, including specialist sexual assault/abuse services if indicated. 